\title{
Fluorescence-guided thoracic surgery
}

\author{
Shota Nakamura^, Masaki Goto, Toyofumi F. Chen-Yoshikawa \\ Department of Thoracic Surgery, Nagoya University Graduate School of Medicine, Nagoya, Japan \\ Contributions: (I) Conception and design: S Nakamura, TF Chen-Yoshikawa; (II) Administrative support: TF Chen-Yoshikawa; (III) Provision of \\ study materials or patients: S Nakamura; (IV) Collection and assembly of data: S Nakamura; (V) Data analysis and interpretation: S Nakamura; (VI) \\ Manuscript writing: All authors; (VII) Final approval of manuscript: All authors. \\ Correspondence to: Toyofumi F. Chen-Yoshikawa, MD. Department of Thoracic Surgery, Nagoya University Graduate School of Medicine, 65 \\ Tsurumai-cho, Showa-ku, Nagoya 466-8550, Japan. Email: tyoshikawa@med.nagoya-u.ac.jp.
}

\begin{abstract}
Recently, the use of sublobar resection has become increasingly common for patients that were previously planned to undergo lobectomy. We encountered two challenges when performing sublobar resections for patients with small pulmonary nodules; one challenge was obtaining a sufficient surgical margin and the other was identifying the location of the nodules, especially in minimally invasive surgery. In recent years, indocyanine green (ICG) fluorescence has been used to solve these two problems in thoracic surgery: first, identifying the accurate intersegmental plane in anatomical lung segmentectomy for detecting the regions of residual pulmonary blood flow, and second, displaying the tumor location or optimal resection line intraoperatively as ICG virtual-assisted lung mapping (ICG-VAL-MAP). The purpose of this article is to present a review of the outcomes of fluorescence-guided thoracic surgery, and also our techniques in minimally invasive surgery using ICG fluorescence. We showed that patients received anatomical pulmonary resection that identified the intersegmental or interlobar plane using ICG guidance in video-assisted thoracic surgery (VATS) or in robot-assisted segmentectomy. We also showed that patients received pulmonary wedge resection, displaying the tumor location intraoperatively using ICG. Furthermore, we introduced a technique of preoperative transbronchial lung marking for small lung nodules called ICG-VAL-MAP. Fluorescence guidance in minimally invasive thoracic surgery makes it possible to ensure a safe surgical margin and identify the intersegmental line and location of the nodules accurately.
\end{abstract}

Keywords: Anatomical segmentectomy; fluorescence-guided thoracic surgery; indocyanine green (ICG); minimally invasive thoracic surgery; indocyanine green virtual-assisted lung mapping (ICG-VAL-MAP)

Received: 22 November 2020; Accepted: 07 January 2021; Published: 20 April 2021.

doi: $10.21037 /$ jovs-20-166

View this article at: http://dx.doi.org/10.21037/jovs-20-166

\section{Introduction}

Small pulmonary nodules that could not previously be detected with conventional radiography have become identifiable at earlier and potentially more curable stages because of the introduction of low-dose helical computed tomography (CT) in lung cancer screening $(1,2)$. When planning surgical resection for patients with small nodules, surgeons face several issues, such as obtaining a sufficient surgical margin and identifying the location of the nodules.

For patients with small nodules, anatomical pulmonary segmentectomy has become an increasingly common option instead of lobectomy. Although sufficient surgical margins are available by anatomical segmentectomy compared with wedge resection, the intersegmental plane is at times difficult to identify accurately. An incomplete intersegmental line can result in congestive lung or

^ ORCID: 0000-0002-1865-0867. 


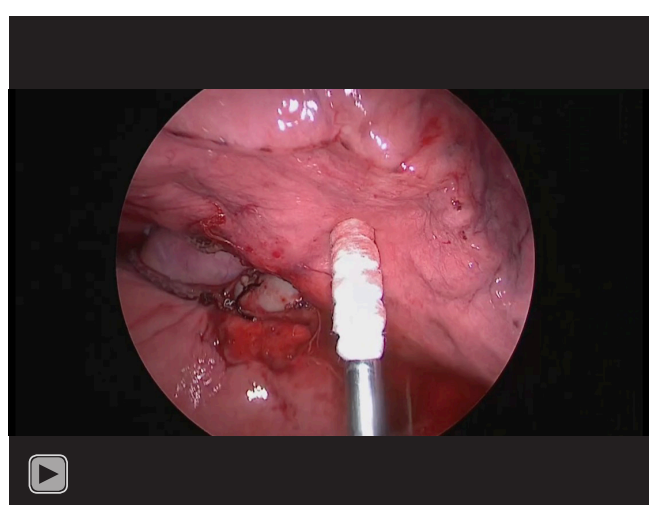

Video 1 The video shows an operation using video-assisted thoracic surgery with a fluorescence imaging camera.

ventilatory mismatch. Additionally, another problem for patients with small nodules was the difficulty of identifying the tumor location. Preoperative or intraoperative marking techniques have become necessary for the resection of nonpalpable small nodules.

In recent years, indocyanine green (ICG) fluorescence has been used to solve the following two problems in thoracic surgery: first, identification of the accurate intersegmental plane in anatomical lung segmentectomy for detecting areas of residual pulmonary blood flow (3-5) and, second, displaying the location of the tumor or optimal resection line intraoperatively as ICG virtual-assisted lung mapping (ICG-VAL-MAP) (6). Herein, we introduce images and videos that describe the features of surgery using the new ICG technology in the field of minimally invasive thoracic surgery.

\section{Identification of the intersegmental or interlobar plane}

\section{Case 1: ICG guidance in video-assisted thoracic surgery (VATS) anatomical segmentectomy}

The present case had one nodule on the left side of the lung; the tumor was a metastatic lung tumor in the central position of S6, measuring $0.7 \mathrm{~cm}$ in size. We planned left S6 anatomical segmentectomy for the patient. An operation was performed with a 3-port VATS. Ligation and dissection of the corresponding bronchovascular branch to the S6 segment were performed in the beginning. Subsequently, ICG was reconstituted using distilled water to prepare a $2-\mathrm{mg} / \mathrm{mL}$ solution, and $3 \mathrm{~mL}$ was injected using a peripheral intravenous catheter; next, $10 \mathrm{~mL}$ of sterile normal saline was added. We used infrared endoscopic equipment. The scope was $10 \mathrm{~mm}$ in diameter and had a $30^{\circ}$ rigid scope. After the change from white-light mode to ICG mode using a foot control panel, the surgical field was visualized with the integrated fluorescence imaging capability. The target segment, which was isolated from the pulmonary vasculature, showed no fluorescence and was dark. The remaining portion of the lung, perfused with ICG, turned fluorescent blue. Subsequently, we identified the true intersegmental plane as the line that separated the dark and blue lung parenchyma. Next, the true plane was marked using cautery and stapled across to complete the lung parenchyma division (Video 1). The postoperative course of the patient was event-free, and the surgical margin distance between the tumor and staple line was $2.1 \mathrm{~cm}$.

\section{Case 2: ICG guidance in robot-assisted segmentectomy}

The present case had two nodules on the right side of the lung; the main tumor was present in the middle lobe, diagnosed as a typical carcinoid tumor measuring $1.5 \mathrm{~cm}$ in size (Figure 1A), and another in the central position of the S8 segment, measuring $0.7 \mathrm{~cm}$ in size, without a confirmed histopathological diagnosis (Figure 1B). We planned an S8 anatomical segmentectomy with a middle lobectomy for complete resection. High-resolution computed tomography (HRCT) was performed preoperatively. Using the HRCT data, we performed a three-dimensional simulation using simulation software (Volume Analyzer Synapse Vincent; Fujifilm Medical Systems, Tokyo, Japan). The simulation workstation allowed us to create a virtual intersegmental plane after plotting the dominant segmental pulmonary artery, thus allowing proper preoperative planning for the patient (Figure 1C). An operation was performed on the Da Vinci Xi (Intuitive Surgical, Sunnyvale, Calif) robot platform using the completely portal 4-arm approach and a near-infrared vision (NIF) light source, Firefly fluorescence imaging camera (Intuitive Surgical). The ligation and dissection of the corresponding bronchovascular branch to the S8 segment and middle lobe were performed in the beginning. After intravenous injection of ICG, the mode of monitoring was changed instantly from the white-light mode to the Firefly mode by using a hand control panel. We used infrared endoscopic equipment that is widely available in contemporary da Vinci robot systems (Intuitive Surgical Systems, Sunnyvale, CA, USA, Firefly in the DaVinci Xi). The scope was $10 \mathrm{~mm}$ in diameter and had a $30^{\circ}$ rigid scope. The surgical field was visualized with the integrated 

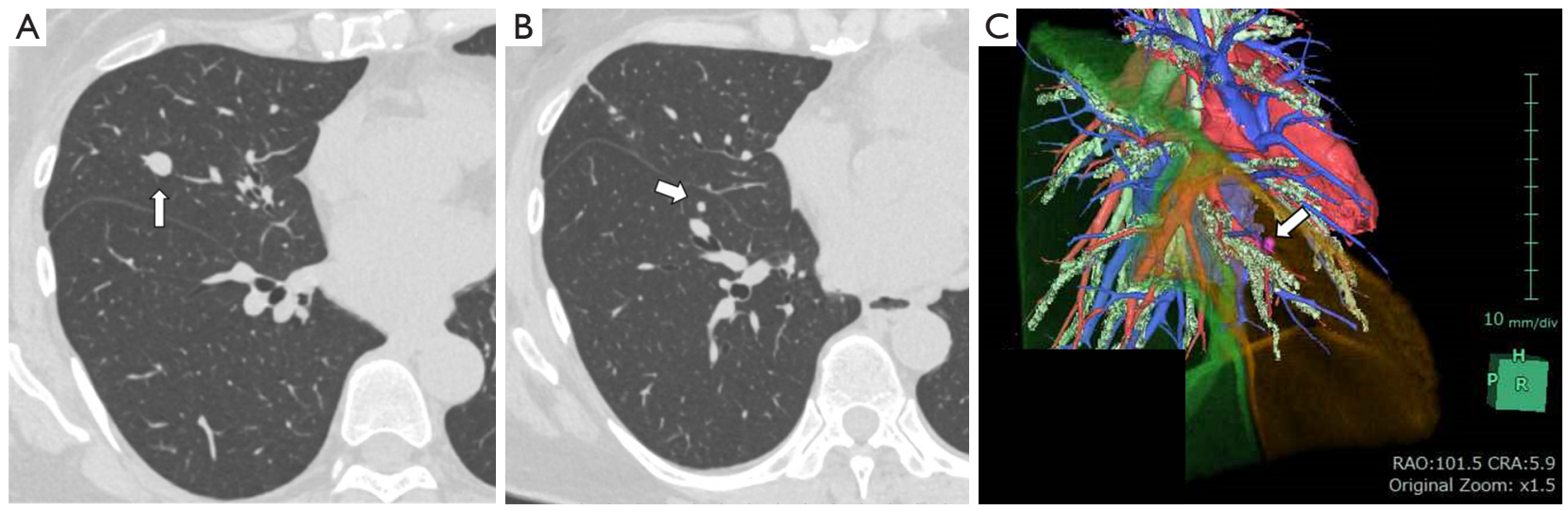

Figure 1 Images of the computed tomography before surgery. (A) A main tumor in the middle lobe diagnosed as a typical carcinoid tumor measuring $1.5 \mathrm{~cm}$ in size (white arrow). (B) Another tumor in the central position of the S8 segment measuring $0.7 \mathrm{~cm}$ in size, without a confirmed histopathological diagnosis (white arrow). (C) Preoperative three-dimensional simulation of the intersegmental bronchus, bronchovascular branch, and intersegmental line using simulation software (Volume analyze Synapse Vincent, Fujifilm Medical Systems, Tokyo, Japan) for S8 anatomical segmentectomy. The arrow shows small nodule in the right S8 segment.

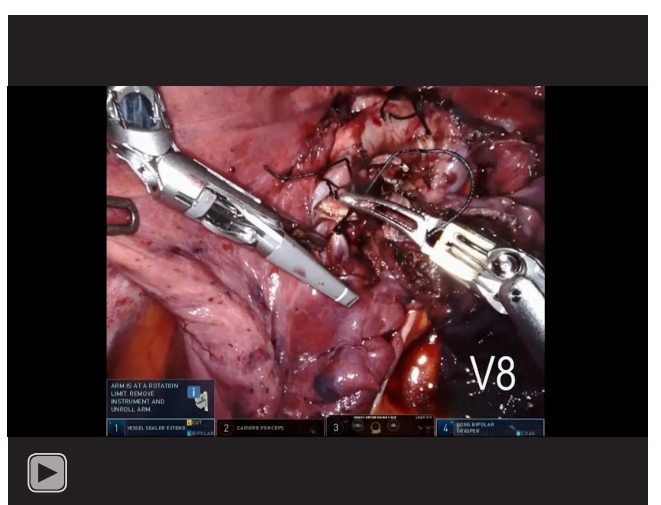

Video 2 The video shows an operation on the Da Vinci Xi robot platform using a Firefly fluorescence imaging camera.

fluorescence imaging capability of the Firefly camera. The target segment, which was isolated from the pulmonary vasculature, showed no fluorescence and was dark. The remaining portion of the lung, perfused with ICG, turned fluorescent green. The true intersegmental plane was then identified as the line separating the dark lung parenchyma from the green lung parenchyma. The true plane was then marked by cautery and stapled across to complete the division of the lung parenchyma. The middle lobe and S8 segment were resected en bloc, not separately (Video 2). The postoperative course of the patient was event-free, and the surgical margin distance between the tumor and the staple line was $1.2 \mathrm{~cm}$.

\section{Case 3: identification of the interlobar plane in robot- assisted lobectomy}

For the present patient who had a pulmonary metastasis in the middle lobe, a right middle lobectomy was planned. The tumor was near the upper lobe (minor fissure), and the patient has an incomplete interlobar line; therefore, the interlobar plane must be accurately identified to obtain sufficient surgical margin (Figure 2). The operation was performed on the Da Vinci Xi (Intuitive Surgical, Sunnyvale, Calif) robot platform using the completely portal 4-arm approach and a NIF light source, Firefly fluorescence imaging camera (Intuitive Surgical). In the beginning, the ligation and dissection of the corresponding bronchovascular branch to the middle lobe was performed. After the ICG injection, the surgical field was visualized with the integrated fluorescence imaging capability of the Firefly camera. The true interlobar plane was then identified as the line separating the dark lung parenchyma from the green lung parenchyma. The true plane was then marked by cautery and stapled by cutting into the right upper lobe largely beyond the marked interlobar line (minor fissure) to ensure a sufficient surgical margin (Video 3). The postoperative course of the patient was event-free, and the surgical margin distance between the tumor and the staple line was $2.5 \mathrm{~cm}$. 


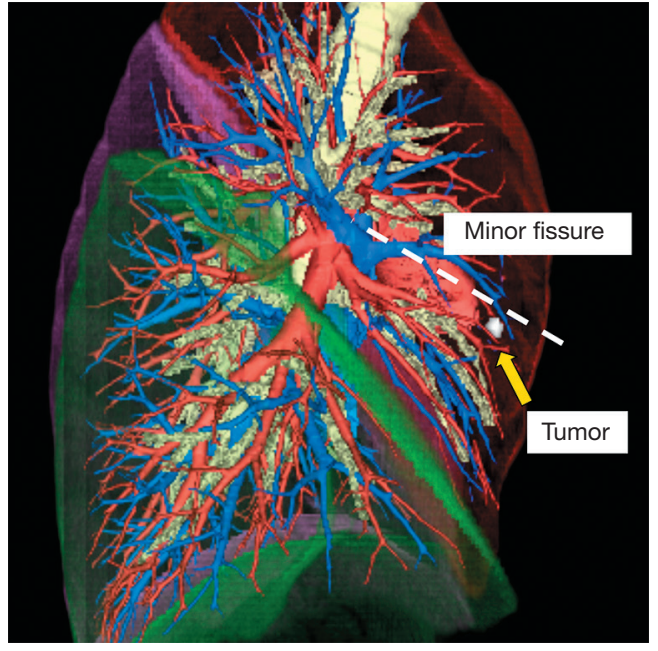

Figure 2 The image shows a preoperative three-dimensional simulation of the lobar bronchus, bronchovascular branch, and interlobar line using simulation software (Volume analyze Synapse Vincent; Fujifilm Medical Systems, Tokyo, Japan) for the right middle lobectomy. The tumor was near the upper lobe (minor fissure). The yellow arrow shows the location of the tumor.

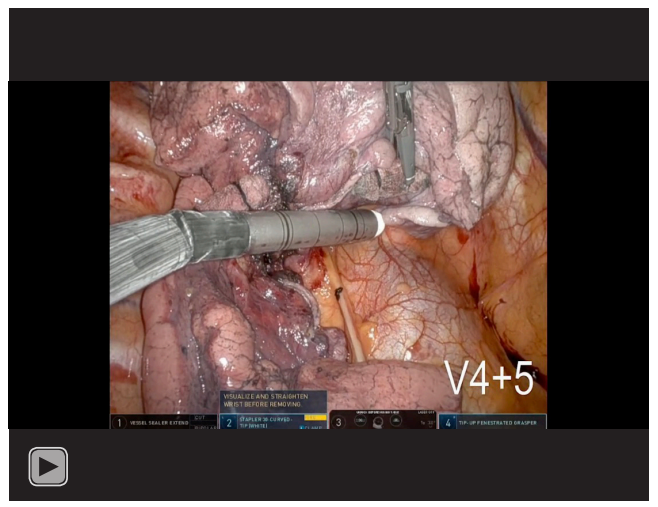

Video 3 The video shows an operation on the Da Vinci Xi robot platform using a Firefly fluorescence imaging camera. A right middle lobectomy was performed with a sufficient surgical margin.

\section{Display of the tumor location intraoperatively using ICG}

\section{Case 4: identification of the tumor location in pulmonary metastasectomy}

The present patient was diagnosed of having a recurrent hepatoblastoma with solitary pulmonary metastasis. The tumor was identified in the left lower lobe. In this patient, ICG was applied during the resection of the metastatic

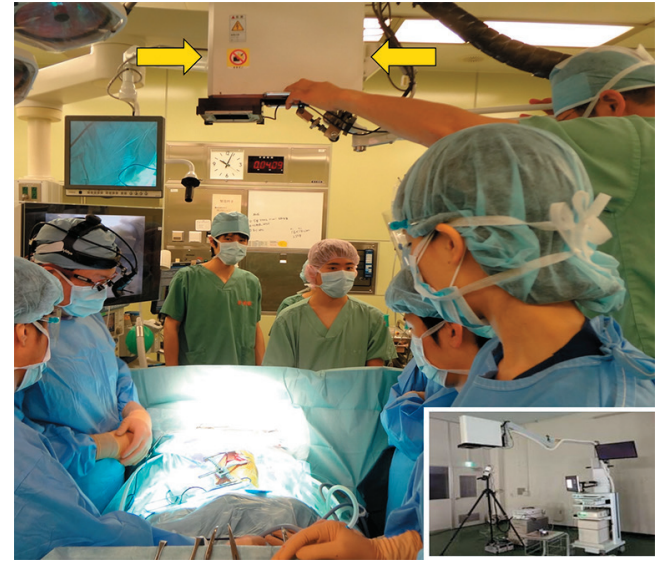

Figure 3 The images show the MIPS. At 24 hours before the surgery, ICG was administered intravenously. In the left hemithorax, the projection mapping technique clearly revealed the lesion. The MIPS showed the real-time fluorescent image on the lung surface during the surgery. The arrows show the medical imaging projection imaging system. MIPS, Medical Imaging Projection System; ICG, indocyanine green.

lesion using the Medical Imaging Projection System (MIPS; Figure 3). Moreover, 24 hours before the surgery, ICG was administered intravenously at a dosage of $0.5 \mathrm{mg} / \mathrm{kg}$. Intraoperatively, the projection mapping technique revealed the lesion clearly, and no other lesions were detected in the left hemithorax. The MIPS projected the real-time fluorescent image on the surface of the lung during the surgical procedure (Figure 4). A left basal segmentectomy was performed with the guidance of ICG fluorescent imaging. The MIPS confirmed that no remnant tumor remained after the resection. The cut surface of the tumor also emitted ICG fluorescence.

\section{Preoperative transbronchial lung marking for small lung nodules}

Cases 5 and 6: VATS anatomical segmentectomy for small lung cancers

VAL-MAP was developed by a Japanese group to identify small pulmonary nodules during surgery (7). In the last decade, the use of VAL-MAP as a standard procedure in daily practice has spread in Japan. However, it has several drawbacks, such as difficulty in dye identification on postmapped preoperative CT (Figure $5 A$ ) and during surgery (Figure $5 B$ ). To solve this problem, a new method, ICG- 
VCL-MAP, was developed by Chen-Yoshikawa et al. (6). In ICG-VAL-MAP, a virtual bronchoscopy navigation system (Synapse Vincent; Fujifilm Medical, Tokyo, Japan) was used to identify the target bronchi that would reach the designed marking points and to plan bronchoscopic procedures. Three makings were set to indicate the lesion for the present patient. After planning with a virtual bronchoscopy, an actual bronchoscopy was performed under sedation and local anesthesia on the day, before the surgery. For

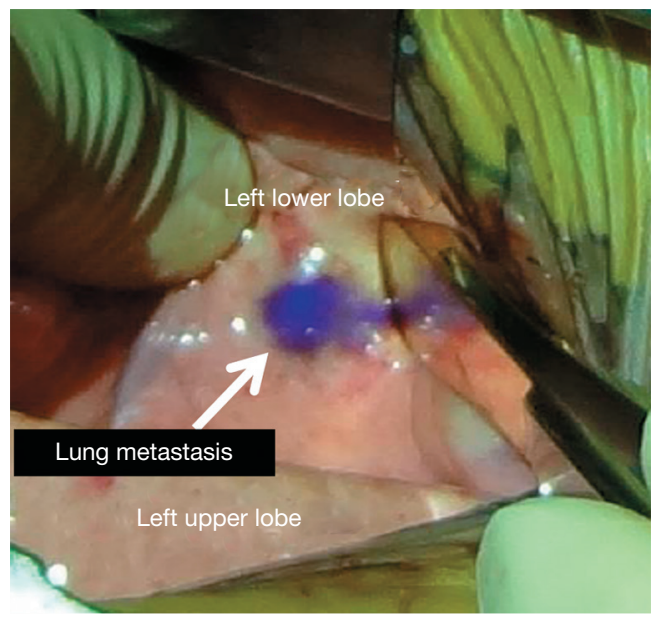

Figure 4 The image shows the nodule of the left lower lobe that was detected using the MIPS. The MIPS revealed the real-time fluorescent image on the lung nodule surface during the metastasectomy for hepatoblastoma. MIPS, Medical Imaging Projection System. The white arrow shows the lung metastatic nodule. better visualization, ICG in addition to a contrast agent, instead of indigo carmine, was injected while confirming fluoroscopically that the tip of the catheter had reached the visceral pleura. Thereafter, we performed another CT scan after the mapping to confirm the location of markings. The CT image after ICG-VAL-MAP was reassembled to form three-dimensional images, including the target marking and lesion. A wide wedge resection was performed using VATS. One patient (Case 5) had a small solid nodule in the right upper lobe (Figure 6A). Preoperatively, the tumor was marked using VAL-MAP (indigo carmine) and ICG-VAL-MAP (ICG and contrast agent; Figure 6B). Nearinfrared endoscopy was used to visualize the ICG markings. Intraoperatively, the marking was easily identifiable in green on near-infrared thoracoscopy, and the tumor was resected using VATS with sufficient surgical margin (Video 4). The tumor was diagnosed pathologically as an adenocarcinoma. The other patient (Case 6) had a small nodule in the right upper lobe, which was planned for S2 anatomical segmentectomy. Preoperative mapping (ICG-VAL-MAP) was performed transbronchoscopically (Figure 7). Under the guidance of ICG mapping, the tumor was resected using VATS right S2 segmentectomy with a sufficient surgical margin and diagnosed pathologically as adenocarcinoma (Video 5).

\section{Discussion}

For patients with small lung nodules, intentional sublobar resection is considered on the basis of the recent evidence $(6,8)$. Furthermore, out of the increasing number of patients that
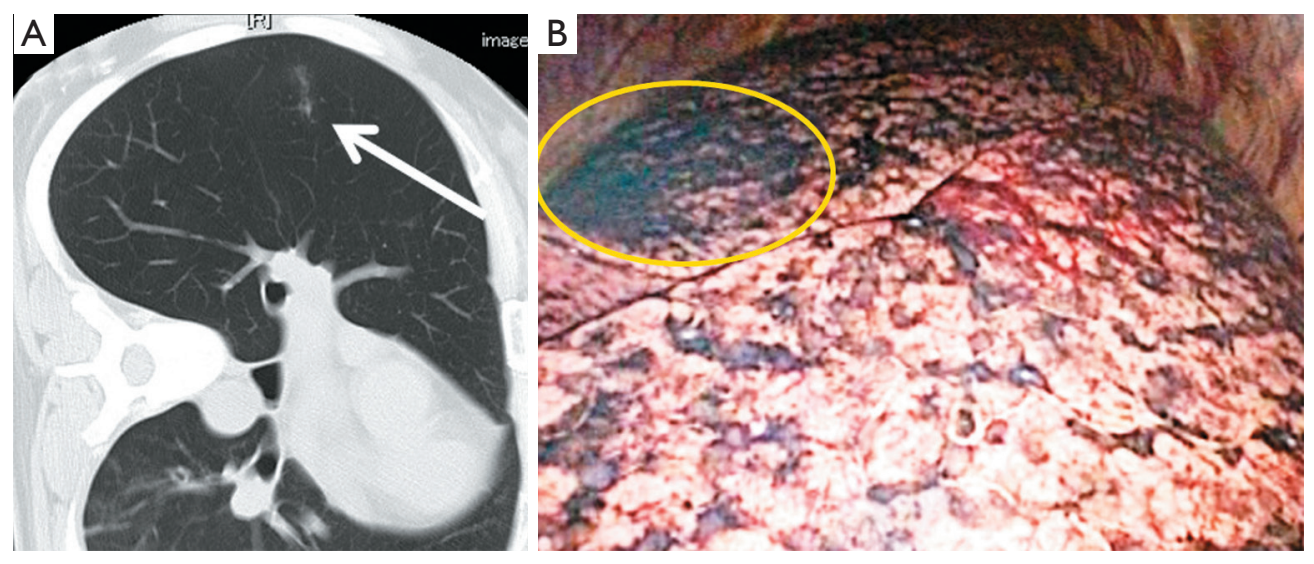

Figure 5 A case with small pulmonary nodule. (A) Post-mapped preoperative CT image obtained with VAL-MAP. The marked dye is difficult to identify. The arrow indicates the location marked by VAL-MAP on CT image. (B) The image shows the left chest cavity and the colored surface of the lung with severe anthracosis. The marked dye is difficult to identify. These difficulties sometimes occur in VAL-MAP. The yellow oval indicates the location marked by VAL-MAP. VAL-MAP, virtual-assisted lung mapping. 


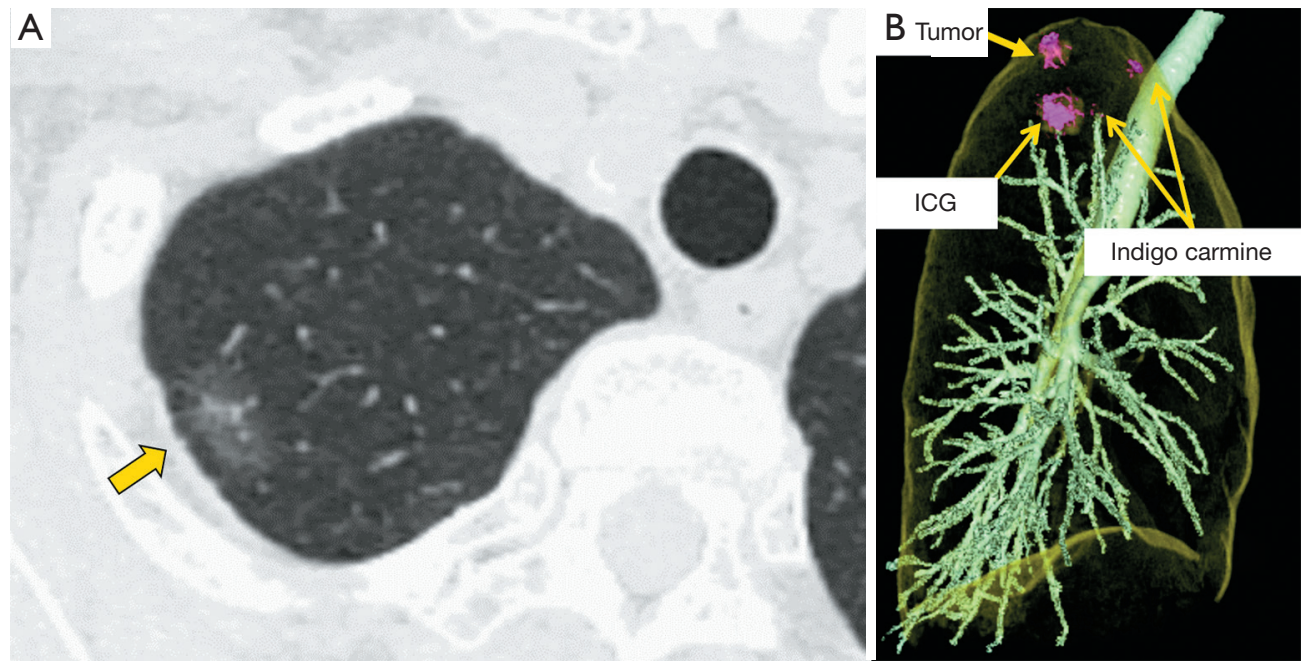

Figure 6 A case with part-solid nodule in right upper lobe. (A) Preoperative CT images. The target nodule is located in the right upper lobe and shown as ground-glass opacity lesion. The arrow shows the location of the target nodule. (B) Three-dimensional image reconstructed using CT data taken after ICG-VAL-MAP, showing the target lesion and marking. The arrows show the locations of target tumor and marker by ICG-VAL-MAP. CT, computed tomography; ICG, indocyanine green; VAL-MAP, virtual-assisted lung mapping.

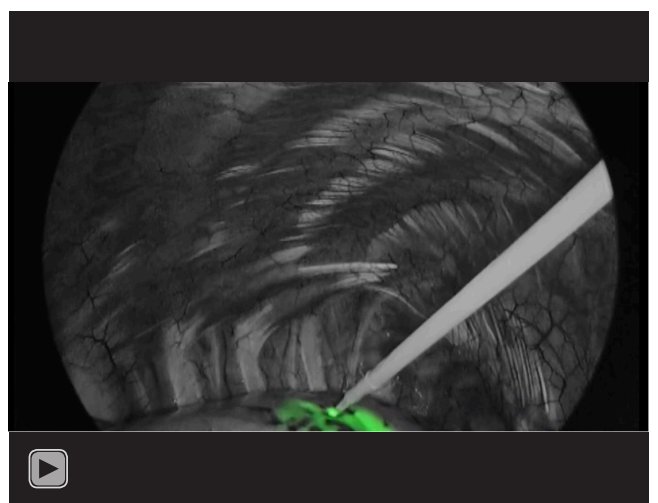

Video 4 The intraoperative process in a case of wide wedge resection of a right upper nodule using video-assisted thoracic surgery.

gain long-term survival after initial surgery, some have opportunities to receive a second surgery. These patients who are indicated to undergo a second surgery or had severe comorbidities, are given the option to undergo a sublobar resection for negative reasons. In recent years, sublobar resection has become an increasingly common option for cases previously planned for lobectomy. We encountered two issues when performing sublobar resections for patients with small pulmonary nodules, of which, one was obtaining a sufficient surgical margin and the other was identifying the location of the nodules, especially in minimally invasive surgery. To solve these two problems, a new surgical technique using ICG fluorescence was introduced (6).

ICG has been widely used in surgery for several organs (i.e., hepatic segmentectomies). In the thoracic field, ICG was used for identifying sentinel lymph nodes or tumor localization initially (3-5). With regard to conditions in the esophagus, ICG is used to prevent the complication of chylothorax during esophagectomy, which is performed to identify the thoracic duct (9). In the mediastinum, although identification of mediastinal tumors or lymph node metastases is attempted, these techniques remain in the basic experimental stage and have not yet been used in daily practice $(10,11)$. While a large uptake of ICG was reported in pulmonary metastases such as hepatoblastoma (12), ICG uptake is generally recognized to be nonuniform in pulmonary metastases from other organs (13). Thereafter, ICG for thoracic surgery was introduced as a technique to detect organ blood flow (14).

Traditionally, when thoracic surgeons detect the intersegmental plane, they used two intraoperative tools, visual inspection and manual palpation, and target segmental inflation to detect the intersegmental plane. However, in patients with emphysema, the intersegmental line is often difficult to determine precisely. Furthermore, when the surgical view is limited, such as during minimally invasive surgery; the inflated lung could affect the procedure. As an alternative to these conventional 
CT; post-ICG-VAL-MAP

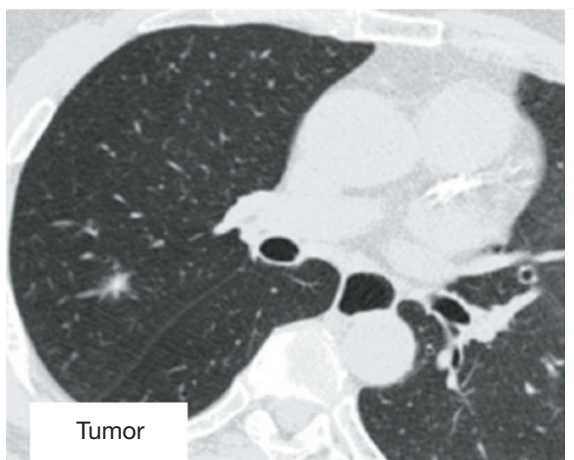

marked by indigo carmine, ICG and contrast agent

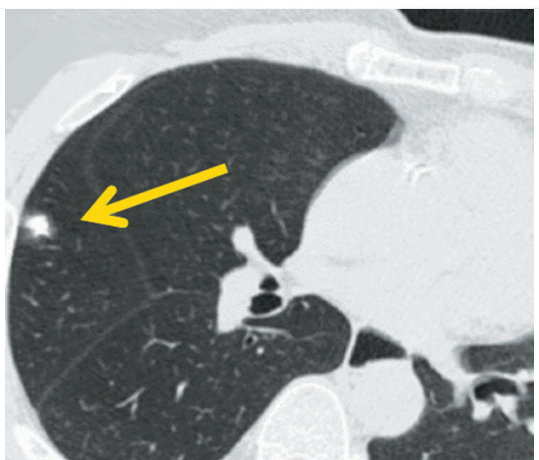

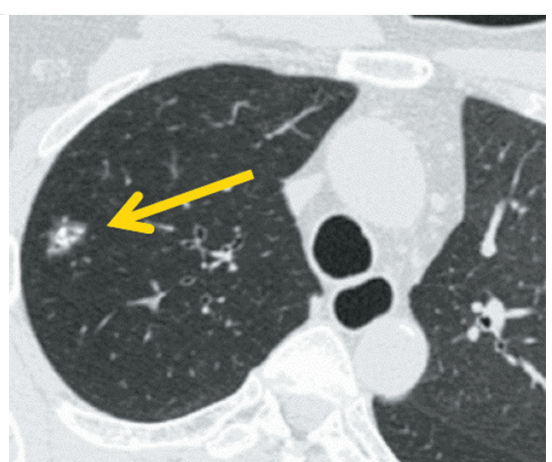

Figure 7 Preoperative CT images. The target nodule is located in the right upper lobe. Preoperative mapping by ICG-VAL-MAP was performed, and the markers are located in the intersegmental plane using ICG and contrast agent. The arrows indicate locations of marker by ICG-VAL-MAP. CT, computed tomography; ICG, indocyanine green; VAL-MAP, virtual-assisted lung mapping.

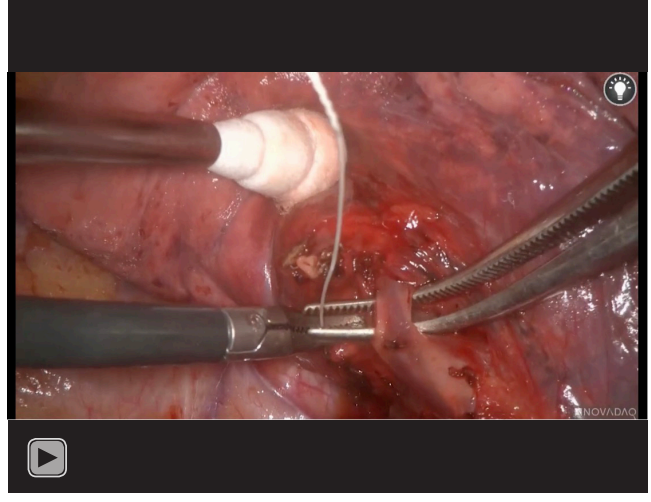

Video 5 Intraoperative process of a case of S2 anatomical segmentectomy by video-assisted thoracic surgery for a right upper nodule.

methods, the ICG technique introduced herein on the basis of our experiences is extremely useful for identifying the intersegmental or interlobar plane accurately, with advantages in minimally invasive pulmonary resection (14-16). Using preoperative three-dimensional simulation combined with ICG technique, we could perform a safe and curative anatomical segmentectomy under VATS or robot-assisted surgery. Furthermore, in this review, we present a video of our ingenuity in identifying the interlobar plane accurately using ICG in patients with invisible interlobar lines.

ICG-VAL-MAP has evolved as a tool for thoracic surgeons to use in the resection of peripheral small nodules. Various methods have been developed to help identify the location of such small nodules $(6,17)$. However, conventional marking techniques, such as CT-guided percutaneous marking using a hook wire, have serious complications, including pneumothorax, hemorrhage, and the potentially fatal air embolism $(18,19)$. These complications could be avoided using ICG-VAL-MAP. This technique is the latest technology that can be applied in VAL-MAP procedures. Although VAL-MAP plays important roles in navigation and localization for the lung resection $(20,21)$, identification of the marking on CT images after VAL-MAP is sometimes difficult. Failure to identify the markings would make lung resection planning challenging, including setting the cutting lines and ensuring sufficient surgical margin. To overcome this weak point, VAL-MAP was revised as ICGVAL-MAP and developed to add a CT contrast to the dye agent. ICG-VAL-MAP was superior in terms of visibility on preoperative CT and providing accurate information intraoperatively. The method enabled surgeons to perform a safe and reliable resection of small nodules.

In the future, we can expect that tumor cells will be marked using ICG navigation (22). Establishment of this method will enable the detection of tumor localization and identification of the presence of lymph node metastases and lymphatic network. It will also allow better lung preservation without compromising the curability as compared with the current standard procedures.

\section{Conclusions}

With fluorescence-guided thoracic surgery in minimally invasive surgery, a safe surgical margin length could be obtained and the segmental line and tumor location could be accurately identified. 


\section{Acknowledgments}

Funding: None.

\section{Footnote}

Peer Review File: Available at https://jovs.amegroups.com/ article/view/10.21037/jovs-20-166/prf

Conflicts of Interest: All authors have completed the ICMJE uniform disclosure form (available at https://jovs. amegroups.com/article/view/10.21037/jovs-20-166/coif). The authors have no conflicts of interest to declare.

Ethical Statement: The authors are accountable for all aspects of the work in ensuring that questions related to the accuracy or integrity of any part of the work are appropriately investigated and resolved. All procedures performed in this study were in accordance with the Helsinki Declaration (as revised in 2013). The manuscript is waived from patient informed consent according to the ethics committee or institutional review board.

Open Access Statement: This is an Open Access article distributed in accordance with the Creative Commons Attribution-NonCommercial-NoDerivs 4.0 International License (CC BY-NC-ND 4.0), which permits the noncommercial replication and distribution of the article with the strict proviso that no changes or edits are made and the original work is properly cited (including links to both the formal publication through the relevant DOI and the license). See: https://creativecommons.org/licenses/by-nc-nd/4.0/.

\section{References}

1. Sone S, Takashima S, Li F, et al. Mass screening for lung cancer with mobile spiral computed tomography scanner. Lancet 1998;351:1242-5.

2. Henschke CI, McCauley DI, Yankeleviltz DF, et al. Early lung cancer action project: overall design and findings from baseline screening. Lancet 1999;354:99-105.

3. Kasai Y, Tarumi S, Chang SS, et al. Clinical trial of new methods for identifying lung intersegmental borders using infrared thoracoscopy with indocyanine green: comparative analysis of 2- and 1-wavelength methods. Eur J Cardiothorac Surg 2013;44:1103-7.

4. Okusanya OT, Hess NR, Luketich JD, et al. Infrared intraoperative fluorescence imaging using indocyanine green in thoracic surgery. Eur J Cardiothorac Surg 2018;53:512-8.

5. Rho J, Lee JW, Quan YH, et al. Fluorescent and Iodized emulsion for preoperative localization of pulmonary nodules. Ann Surg 2019. doi: 10.1097/ SLA.0000000000003300.

6. Chen-Yoshikawa TF, Fukui T, Nakamura S, et al. Current trends in thoracic surgery. Nagoya J Med Sci 2020;82:161-74.

7. Sato M, Omasa M, Chen F, et al. Use of virtual assisted lung mapping (VAL-MAP), a bronchoscopic multisport dye-marking technique using virtual images, for precise navigation of thoracoscopic sublobar lung resection. J Thorac Cardiovasc Surg 2014;147:1813-9.

8. Suzuki K, Saji H, Aokage K, et al. Comparison of pulmonary segmentectomy and lobectomy: Safety results of a randomized trial. J Thorac Cardiovasc Surg 2019;158:895-907.

9. Van Daele E, Van Nieuwenhove Y, Ceelen W, et al. Nearinfrared fluorescence guided esophageal reconstructive surgery: A systematic review. World J Gastrointest Oncol 2019;11:250-63.

10. Zhao S, Guo X, Taniguchi M, et al. Detection of mediastinal lymph node metastases using indocyanine green (ICG) fluorescence imaging in an orthotopic implantation model. Anticancer Res 2020;40:1875-82.

11. Predina JD, Keating J, Newton A, et al. A clinical trial of intraoperative near-infrared imaging to assess tumor extent and identify residual disease during anterior mediastinal tumor resection. Cancer 2019;125:807-17.

12. Chen-Yoshikawa TF, Hatano E, Yoshizawa A, et al. Clinical application of projection mapping technology for surgical resection of lung metastasis. Interact Cardiovasc Thorac Surg 2017;25:1010-1.

13. Hamaji M, Chen-Yoshikawa TF, Minami M, et al. NearInfrared Imaging Using Intravenous Indocyanine Green at a Conventional Dose to Locate Pulmonary Metastases: A Pilot Study. Thorac Cardiovasc Surg 2019;67:688-91.

14. Misaki N, Chang SS, Igai H, et al. New clinically applicable method for visualizing adjacent lung segments using an infrared thoracoscopy system. J Thorac Cardiovasc Surg 2010;140:752-6.

15. Pardolesi A, Veronesi G, Solli P, et al. Use of indocyanine green to facilitate intersegmental plane identification during robotic anatomic segmentectomy. J Thorac Cardiovasc Surg 2014;148:737-8.

16. Mehta M, Patel YS, Yasufuku K, et al. Near-infrared mapping with indocyanine green is associated with an 
increase in oncological margin length in minimally invasive segmentectomy. J Thorac Cardiovasc Surg 2019;157:2029-35.

17. Nakamura S, Hayashi Y, Kawaguchi K, et al. Clinical application of a surgical navigation system based on virtual thoracoscopy for lung cancer patients: real time visualization of area of lung cancer before induction therapy and optimal resection line for obtaining a safe surgical margin during surgery. J Thorac Dis 2020;12:672-9.

18. Sakiyama S, Kondo K, Matsuoka H, et al. Fatal air embolism during computed tomography-guided pulmonary marking with a hook-type marker. J Thorac Cardiovasc Surg 2003;126:1207-9.

doi: 10.21037/jovs-20-166

Cite this article as: Nakamura S, Goto M, Chen-Yoshikawa TF. Fluorescence-guided thoracic surgery. J Vis Surg 2021;7:18.
19. Horan TA, Pinhreiro PM, Araujo LM, et al. Massive gas embolism during pulmonary nodule hook wire localization. Ann Thorac Surg 2002;73:1647-49.

20. Sato M, Yamada T, Menju T, et al. Virtual-assisted lung mapping: outcome of 100 consecutive cases in a sing le institute. Eur J Cardiothoracic Surg 2015;47:2131-9.

21. Chen-Yoshikawa TF, Date H. Update om three-dimensional image reconstruction for preoperative simulation in thoracic surgery. J Thorac Dis 2016;8:S295-301.

22. Predina JD, Newton AD, Xia L, et al. An open label trial of folate receptor-targeted intraoperative molecular imaging to localize pulmonary squamous cell carcinomas. Oncotarget 2018;9:13517-29. 